\title{
Investigation on Micro-hardness with Shear Strain Extent for Copper Specimens subjected to High Pressure Torsion
}

\author{
Yuan Liu ${ }^{* a}$, Youshi Hong ${ }^{\mathrm{b}}$, Keke Fan ${ }^{\mathrm{a}}$, Xiaodong $\mathrm{Ji}^{\mathrm{c}}$ \\ a The High School Affiliated to Renmin University of China, Beijing 100080, China \\ b Institute of Mechanics, Chinese Academy of Sciences, Beijing 100080, China \\ c School of soil and water conservation, Beijing Forestry University, Beijing, 100083, China
}

\begin{abstract}
High Pressure Torsion (HPT) is an effective way to diminish the size of the crystal particles and improve the mechanical performance of metal material. In this study, the micro-hardness, size of crystal particles and shear strain of copper samples treated with HPT and their relationship were investigated. The results showed that the micro-hardness of the HPT copper increases from 50 to 140 when the nominal shear strain increases from 0 to 5 . Then it reached saturation state and the micro-hardness didn't increase obviously when the shear strain increased further. The grain size becomes smaller as the distance away from the centre of sample for the sample with fewer turns of torsion. For the sample with more turns of torsion, in addition to the grain size refinement, the micro-hardness reaches saturation and its spatial distribution is uniform both along thickness and radius direction.
\end{abstract}

Keywords: Micro-hardness, copper, shear strain, High Pressure Torsion (HPT)

\section{INTRODUCTION}

In general, the mechanical properties of metallic materials may be upgraded with the refinement of their grain size. So, making grain size smaller is one of the effective ways to improve the mechanical performance of metallic materials. Severe Plastic Deformation (SPD) technique is a newly developed method to refine grain size for metallic materials and has great potential for engineering application. High Pressure Torsion (HPT) is a typical method of SPD. In recent years, HPT investigations have been performed for copper specimens, which used mostly the thin specimens and mainly investigated the distribution of shear strain and micro-hardness along the radial direction of specimen. However, there is a lack of investigations on the propensity of shear strain extent and micro-hardness along the thickness direction of HPT specimen.

In this investigation, an experiment is carried out to measure the micro-hardness distribution along both the thickness and the radial direction of HPT copper specimens with $3.5 \mathrm{~mm}$ thickness and $12 \mathrm{~mm}$ diameter, which were produced by a relevant research group in the Institute of Mechanics, Chinese Academy of Sciences. The correlation between microhardness and shear strain extent is demonstrated and analyzed.

\section{TEST PROCEDURE}

\subsection{Preparation of the sample}

There are 5 groups of specimens experienced different HPT processes of $0.5,4,8,12$ and 16 turns of torsion, respectively, which covered a shear strain range of $0 \sim 170$. The arrangement of micro-hardness indentation is: along the radial direction from specimen centre with $0.5,1.0,1.5,2.5,3.5,4.5$ and $5.5 \mathrm{~mm}$; and along thickness direction with 0.1 , $0.25,0.75,1.25,1.75,2.25,2.75,3.25$ and $3.4 \mathrm{~mm}$.

*yuanliu2009@gmail.com

ICEM 2008: International Conference on Experimental Mechanics 2008, edited by Xiaoyuan He, Huimin Xie, Yilan Kang, Proc. of SPIE Vol. 7375, 73752Z · (C) 2009 SPIE · CCC code: 0277-786X/09/\$18 · doi: 10.1117/12.839236 


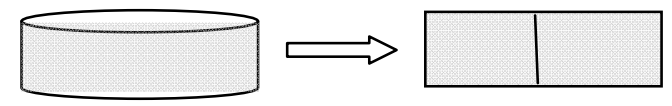

Fig. 1. Schematic of sample cutting

An annealed discal copper sample, with $12 \mathrm{~mm}$ diameter and $3.5 \mathrm{~mm}$ thickness, was installed inside the HPT system shown in Fig.1. In this system, the upper part of the sample is fixed with upper anvil, and the down part of the sample was pressed and twisted by down anvil through the friction between the surfaces of the sample and the down anvil. 5 samples were tested with HPT processes of $0.5,4,8,12$ and 16 turns, respectively. The twisting time is $2-3$ minutes per turn.

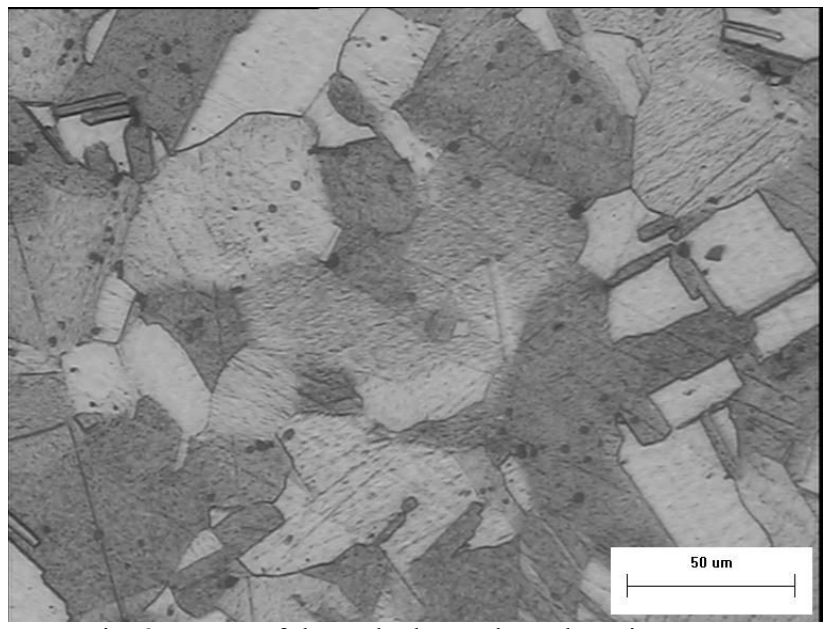

Fig. 2. Image of the etched sample under microscope

As shown in Fig.2 the samples were cut along radial direction using electric spark cutting machine (Type: FL-A1). The shear strain $\gamma$ of the sample can be calculated according to the formula:

$$
\gamma=2 \pi \mathrm{Nr} / \mathrm{h}
$$

where: $\gamma$ : Shear strain
$\mathrm{N}$ : Number of torsion turns
h: Thickness of sample
r: Distance from the torsion center

The shear strain for the 5 test samples ranged from 0 to 172 .

To reveal the microstructure and eliminate the influence of surface layer created during polishing on micro-hardness measurement, the sample was etched with $10 \%$ hyposulphite solution for 40 s before it was observed under microscope.

\subsection{Micro-hardness test}

The distribution of test point on the profile of sample was shown in Fig. 3. The points marked with diamond shape are the main points. Two auxiliary points located on left and right of the main point with $100 \mu \mathrm{m}$ distance, respectively. The average test values of each main point and its two auxiliary points were taken to evaluate the hardness of the main point on the sample profile. 
The micro-hardness indentation was performed according to the distribution of 5 test samples shown in Fig. 4 for. Test condition: load: $50 \mathrm{~g}$; load holding time: $15 \mathrm{~s}$ and temperature: $24^{\circ} \mathrm{C}$.

The Vickers Hardness was calculated by the equation: $\mathrm{Hv}=1.8544 \mathrm{~F} / \mathrm{d}^{2}$

where: $\mathrm{F}$ : load (kgf); d: average length of diagonals of the indentation.

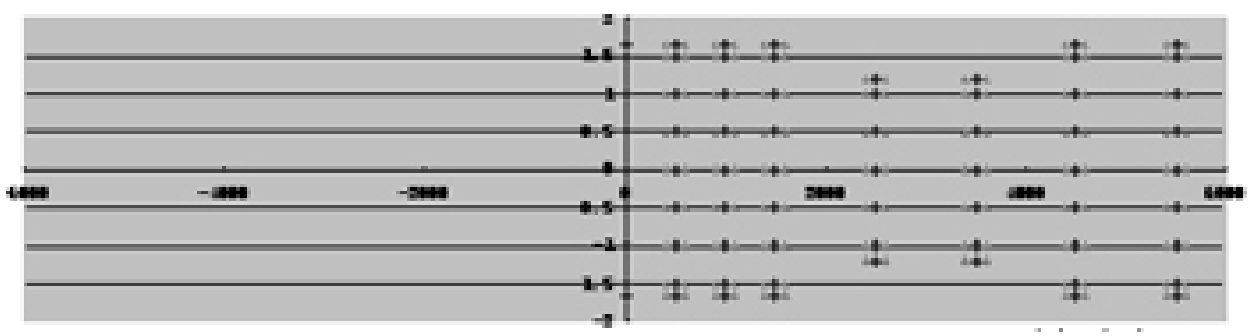

Fig. 3. Micro-hardness indentation distribution on the profile of sample

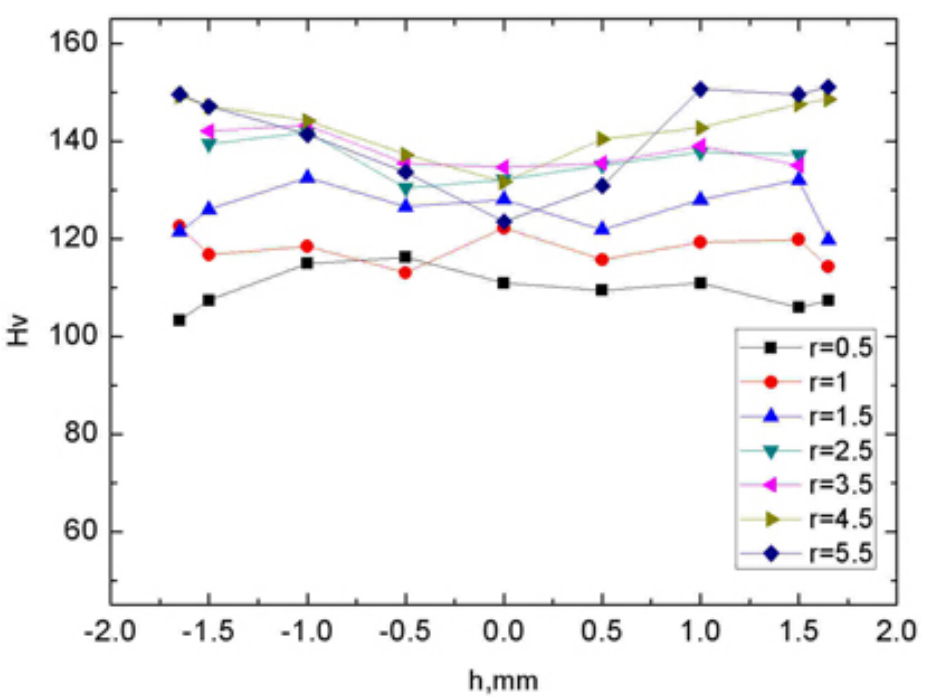

Fig. 4. Micro-hardness distribution along thickness for the sample with 0.5 turns of torsion

\section{RESULTS AND DISCUSSION}

\subsection{Sample with 0.5 turns of torsion}

The results in Fig.4 showed that the micro-hardness values have little difference along the thickness direction when the radius was less than $4.5 \mathrm{~mm}(\mathrm{r} \leq 4.5 \mathrm{~mm})$. When of the radius was more than $4.5 \mathrm{~mm}(\mathrm{r}>4.5 \mathrm{~mm})$, the micro-hardness values varied obviously along the thickness direction, and the values in the middle stage were lower than those in the end. 


\subsection{Relationship between nominal shear}

The nominal shear strain as the function of the micro-hardness was showed in Fig.5. The results showed that the microhardness increased dramatically with the strain increases when the shear strain was relatively small $(\gamma<5)$ In the region near sample edge, the micro-hardness is low in the middle section and high in the two ends along the thickness direction. When the shear strain is moderate $(5<\gamma<100)$, the micro-hardness reaches the saturation state $(300 \%$ of the original value), and its spatial distribution is almost uniform both in radius and thickness direction (standard deviation is less than $5 \%)$. When the shear strain is large $(\gamma>100)$, the micro-hardness increases slightly as the shear strain increases. The micro-hardness spatial distribution is similar to the case for small shear strain, i.e., in the region near sample edge, the hardness is low in the middle section and high in the two ends along the thickness direction. Even under the same nominal shear strain, the micro-hardness of the copper sample processed with HPT may be different if the number of torsion turns is different. This means that the micro-hardness of HPT sample is also related to the loading history. When the nominal strain is between 10 and 100, corresponding to 4 and 8 turns of torsion in this investigation, the distribution of micro-hardness is spatially uniform.

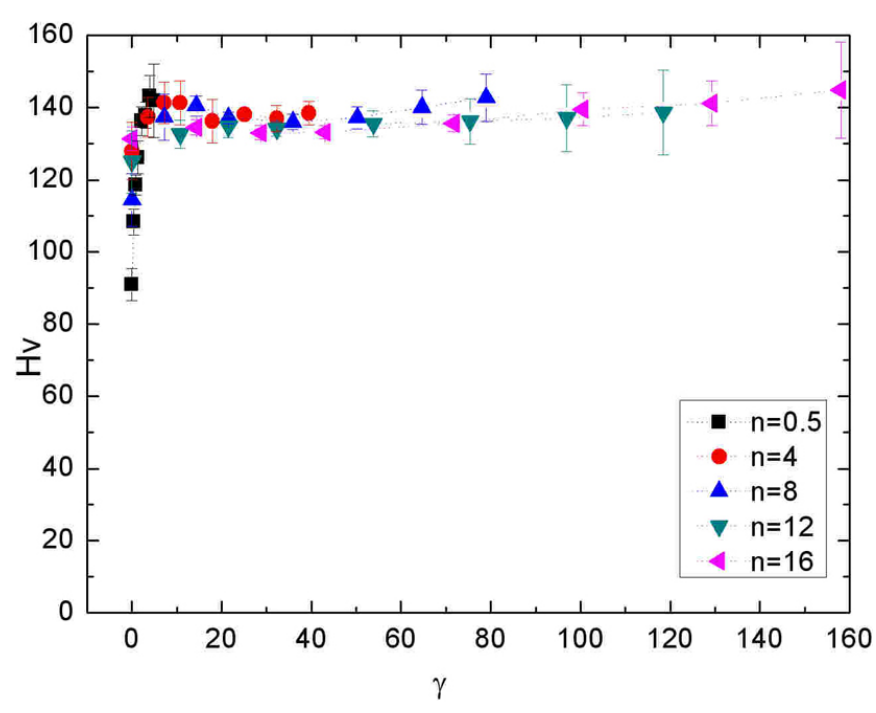

Fig. 5. Variation of nominal shear strain with micro-hardness

\section{CONCLUSION}

(1) For the sample with fewer turns of torsion, the grain size becomes smaller as the distance away from the centre of sample.

(2) For the sample with more turns of torsion, in addition to the grain size refinement, the micro-hardness reaches saturation and its spatial distribution is uniform both along thickness and radius direction. The saturation is the result of the dynamic balance of refinement process of microstructure.

(3) For the samples with 0.5,12 and 16 turns of torsion, in the region near sample edge, the micro-hardness is lower in the middle section and higher in the two ends along the thickness direction. 


\section{REFERENCES}

[1] A.P. Zhilyaev, K. Oh-ishi, T.G. Langdon, T.R. McNelley. Microstructural evolution in commercial purity aluminum during high-pressure torsion. Materials Science and Engineering A 410-411 (2005) 277-280.

[2] A.P. Zhilyaev, G.V. Nurislamova, B.-K. Kim, M.D. Bar'o, J.A. Szpunar,T.G. Langdon, Acta Mater. 51 (2003).

[3] Hong Youshi, Shangguan Fengshou, Xie Ziling. Grain Refinement and Hardness Increase of Copper Subjected to High Pressure Torsion. TMS 136th Annual Meeting \& Exhibition, Orlando, USA, February 2007, p.121.

[4] HONG Youshi, AN Zhongsheng and WANG Huicai, Numerical Simulation for Temperature Field Evolution of Metallic Alloy Surface due to Pulsed Laser Treatment, Key Engineering Materials, (2003), Vols.243-244, p.481486. 